\title{
Conforto no final de vida na terapia intensiva: percepção da equipe multiprofissional
}

End-of-life comfort in intensive care: the perception of the multidisciplinary team Bienestar al final de la vida en unidad de cuidados intensivos: percepción del equipo multiprofesional

Isabella Batista Pires ${ }^{1}$ iD https://orcid.org/0000-0002-1991-5943

Tânia Maria de Oliva Menezes ${ }^{1}$ io https://orcid.org/0000-0001-5819-0570

Bruna Borges de Cerqueira ${ }^{2}$ iD https://orcid.org/0000-0002-6718-4487

Rebeca Santos de Albuquerque ${ }^{3}$ iD https://orcid.org/0000-0003-0720-165X

Halanna Carneiro Guimarães Bastos Moura ${ }^{1}$ id https://orcid.org/0000-0002-7125-1662

Raniele Araújo de Freitas ${ }^{1}$ id https://orcid.org/0000-0002-9367-3639

Alana Libânia de Souza Santos ${ }^{1}$ id https://orcid.org/0000-0002-6503-8742

Emanuela Santos Oliveira ${ }^{1}$ id https://orcid.org/0000-0003-1108-9568

Pires IB, Menezes TM

Como citar:
TM, Cerqueira BB, Albuquerque RS, Moura HC, Freitas RA, et al. Conforto no final de vida na terapia intensiva: percepção da equipe multiprofissional. Acta Paul Enferm. 2020;33:APE20190148.

DOI

http://dx.doi.org/10.37689/actaape/2020A00148

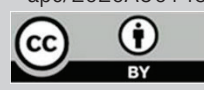

Cuidados paliativos: Conforto do paciente; Unidades de terapia intensiva; Equipe de assistência ao paciente; Teoria de enfermagem

Keywords

Palliative care: Patient confort: Intensive care units; Patient care team; Nursing theory

Descriptores

Cuidados paliativos; Comodidad del paciente; Unidades de cuidados intensivos; Grupo de atención al paciente; Teoría de enfermería

Submetido 17 de Junho de 2019

Aceito

4 de Outubro de 2019

\section{Autor correspondente} Isabella Batista Pires

E-mail: isabellabpires@gmail.com

\section{Resumo}

Objetivo: Analisar a percepção da equipe multiprofissional sobre o conforto no final de vida na terapia intensiva. Métodos: Estudo qualitativo, de caráter descritivo e exploratório, realizado com 50 profissionais da equipe de saúde de uma Unidade de Terapia Intensiva de um hospital privado da Bahia. Utilizou-se um questionário sociodemográfico e a entrevista semiestruturada para coleta de dados, que foram analisados pela técnica de análise de conteúdo temática e discutidos à luz da Teoria do Fim de Vida Pacífico.

Resultados: Os profissionais revelaram que a assistência à saúde de pacientes em cuidados paliativos na Unidade de Terapia Intensiva é direcionada a necessidade da promoção do conforto. Assim, emergiram três categorias: 1. Aliviando a dor para promover conforto; 2 Proporcionando conforto para alcançar paz, dignidade e respeito; $3 \mathrm{~A}$ aproximação com entes queridos e fé como estratégia de conforto.

Conclusão: 0 conforto foi o conceito da Teoria do Final de Vida Pacífico que se destacou na percepção da equipe multiprofissional, sendo promovido por todas as categorias na sua prática assistencial a pacientes em paliação, motivado pela identificação das necessidades básicas desses pacientes. 0 conforto relacionado ao bem-estar físico foi o mais presente nos discursos, sinalizando a necessidade de capacitação multiprofissional para uma assistência do conforto de forma holística. A teoria utilizada foi reconhecida como uma importante ferramenta para subsidiar intervenções que auxiliem na busca por um final de vida pacífico.

\section{Abstract}

Objective: To analyze the perception of the multidisciplinary team about end-of-life comfort in intensive care.

Methods: This is a qualitative, descriptive and exploratory study conducted with 50 professionals of the health team of an Intensive Care Unit of a private hospital in the state of Bahia. A sociodemographic questionnaire and semi-structured interviews were used to collect data, which were analyzed using thematic content analysis technique and discussed in the light of the Peaceful End-of-Life Theory.

Results: Professionals revealed that the health care of patients in palliative care in the Intensive Care Unit is directed to the need to promote comfort. Thus, three categories emerged: 1. Relieving pain to promote comfort; 2. Providing comfort to achieve peace, dignity and respect; 3 . Approaching loved ones and faith as a comfort strategy.

Conclusion: Comfort was the concept of the Peaceful End-of-life Theory that stood out in the perception of the multidisciplinary team. It was promoted by all categories in its care practice to palliative patients, motivated by the identification of the basic needs of these patients. Comfort related to physical well-being was the most 
present in the speeches, signaling the need for multidisciplinary training for holistic comfort assistance. The theory used has been recognized as an important tool to support interventions that help in the search for a peaceful end-of-life.

\section{Resumen}

Objetivo: Analizar la percepción del equipo multiprofesional sobre el bienestar al final de la vida en unidad de cuidados intensivos.

Métodos: Estudio cualitativo, de carácter descriptivo y exploratorio, realizado con 50 profesionales del equipo de salud de una Unidad de Cuidados Intensivos de un hospital privado del estado de Bahia. Se utilizó un cuestionario sociodemográfico y la entrevista semiestructurada para la recolección de datos, que se analizaron con la técnica de análisis de contenido temático y se discutieron de acuerdo con la Teoría Final de Vida Pacífico.

Resultados: Los profesionales revelaron que la asistencia a la salud de pacientes en cuidados paliativos en la Unidad de Cuidados Intensivos se orienta hacia la necesidad de promover el bienestar. De este modo, surgieron tres categorías: 1. Aliviar el dolor para promover el bienestar; 2. Proporcionar bienestar para tener paz, dignidad y respeto; 3. Acercarlos a los seres queridos y a la fe como estrategia de bienestar.

Conclusión: El bienestar fue el concepto de la Teoría Final de Vida Pacífico que se destacó en la percepción del equipo multiprofesional, de forma que todas las categorías lo promueven en la práctica asistencial a pacientes en cuidados paliativos y es motivado mediante la identificación de las necesidades básicas de estos pacientes. El bienestar físico fue el más presente en los discursos, lo que advierte la necesidad de capacitación multiprofesional para brindar asistencia de bienestar de forma holística. La teoría utilizada se reconoce como una importante herramienta para ayudar en las intervenciones que buscan un final de vida pacífico.

\section{Introdução}

A influência do pragmatismo tecnológico e paradigma curativista do modelo biológico na assistência à saúde, principalmente aquela destinada a pacientes em terminalidade, induz à maioria deles finalizar suas vidas na Unidade de Terapia Intensiva (UTI). ${ }^{(1)}$ Nesse sentindo, a equipe multiprofissional vivencia uma mudança do paradigma do cuidado, buscando melhorar a qualidade de vida (QV) daqueles que enfrentam problemas associados a doenças ameaçadoras de vida. Envolve o cuidado do sofrimento além dos sintomas físicos, incluindo o apoio de uma equipe multidisciplinar e o atendimento das necessidades básicas do paciente e sua família. ${ }^{(2)}$

O conforto, associado ao alívio da dor, aproximação com entes queridos, promoção de dignidade e respeito, além da experiência de paz são conceitos da Teoria do Fim de Vida Pacífico (TFVP), que se aproximam com os princípios dos cuidados paliativos (CP). Esta teoria, criada pelas enfermeiras Cornelia Ruland e Shirley Moore, tem por finalidade melhorar a QV e os cuidados ao paciente terminal, relacionado as intervençôes de enfermagem e resultados específicos para esses doentes. ${ }^{(3)}$ Dessa forma, pode ser classificada como uma teoria preditiva e de médio alcance, ${ }^{(4)}$ fornecendo subsídios às práticas assistenciais de $\mathrm{CP}$, com o intuito de proporcionar um final de vida pacífico.

A teoria possui bom alcance na promoção de conforto em ambientes paliativos, possibilitando açóes que contribuam para o encontro da estabilidade do cuidado baseada nos desejos e necessidades básicas dos pacientes. ${ }^{(5)}$ Entretanto, por tratar-se de uma teoria recente, sem testabilidade no Brasil e incipiente no cenário científico, tornam-se necessárias mais publicaçôes, com o intuito de nortear os cuidados ao paciente terminal na UTI. ${ }^{(6)}$

O CP na UTI está diretamente ligado à promoção do conforto. A TFVP denomina a experiência de conforto como alívio do desconforto, o estado de facilidade e contentamento pacífico, o que torna a vida mais fácil ou prazerosa. ${ }^{(3)}$ Diante do crescente número de pacientes com doenças terminais internados nessas unidades e da necessidade de promover um final de vida pacífico para esses indivíduos, utilizou-se como questão norteadora do estudo: Qual a percepção da equipe multiprofissional sobre o conforto no final de vida na terapia intensiva?

O cuidado ao paciente terminal na UTI é complexo, conflitante e desafiador. O modelo biomédico vigente, avanços tecnológicos e enfrentamento frequente da morte são obstáculos comuns, exigindo dos profissionais uma fundamentação do cuidado clínico, a fim de promover QV a esses pacientes. Sendo assim, objetivou-se analisar a percepção da equipe multiprofissional sobre conforto no final de vida na terapia intensiva.

\section{Métodos}

Trata-se de um estudo qualitativo, de caráter descritivo e exploratório, norteado pela ferramenta COREQ, ${ }^{(7)}$ realizado em uma UTI Imunológica, de um hospital particular de grande porte, na cidade 
de Salvador, Bahia. O local de estudo foi definido devido ao elevado número de pacientes em paliação.

Participaram do estudo 50 profissionais: 13 médicos, 13 enfermeiras, nove fisioterapeutas, nove técnicos de enfermagem, três nutricionistas, dois assistentes sociais e um psicólogo. Os critérios de inclusão foram: participantes que já cuidaram de pacientes em CP na UTI; tempo de atuação maior que um ano com paciente crítico e pertencerem ao quadro permanente da unidade. Excluíram-se os profissionais que estavam de férias ou licença médica durante o período da coleta de dados, que ocorreu entre novembro de 2018 a maio de 2019.

A coleta de dados ocorreu através de um questionário de caracterização sociodemográfica e entrevista semiestruturada, realizadas em salas privativas da unidade, previamente agendadas conforme disponibilidade do profissional. Os depoimentos foram gravados em áudio e transcritos na íntegra.

Os profissionais foram entrevistados até atingir a saturaçáo dos dados por categoria. A entrevista foi conduzida pelas questóes: Quais as principais necessidades básicas do paciente em CP na UTI? O que você considera como conforto na assistência aos pacientes em CP?

Os depoimentos foram analisados conforme a técnica de análise de conteúdo de Bardin, através das etapas: 1) pré-análise; 2) exploração do material; 3) tratamento dos resultados e interpretação, ${ }^{(8)}$ discutidos à luz dos conceitos da TFVP.

As diretrizes e normas regulamentadoras de pesquisa com seres humanos, presentes na Resolução 466/12 $2^{(9)}$ foram respeitadas, sendo aprovado pelo Comitê de Ética em Pesquisa do hospital lócus, com parecer $\mathrm{n}^{\circ} 2.890 .509$ e CAAE 93808218.9.0000.0048. Os participantes assinaram o Termo de Consentimento Livre e Esclarecido. Para manutenção do anonimato dos participantes foram identificados pela letra inicial da categoria, seguida da ordem das entrevistas (M01, M02; E01, E02).

\section{Resultados}

Houve predomínio de mulheres (72\%) e adultos jovens (faixa etária de 30 a 45 anos com 70\%), com idade média de 37,5 anos. Quanto à especialização, $56 \%$ possui em UTI e nenhum em CP. Embora todos os participantes afirmaram que já prestaram assistência a pacientes em fase terminal, somente 32\% tiveram abordagem sobre a temática na graduação, destacando-se os enfermeiros, 53,8\%.

A partir da identificação das necessidades básicas do paciente em CP na UTI, os participantes direcionaram o cuidado para os conceitos da TFVP, priorizando a promoção do conforto.

Três categorias emergiram: 1. Aliviando a dor para promover conforto; 2 . O conforto para alcançar paz, dignidade e respeito e 3. A aproximação com entes queridos e fé como estratégia de conforto.

\section{Aliviando a dor para promover conforto}

A TFVP traz como conceito essencial no cuidado ao paciente proporcionar experiência de não sentir dor. Para promover o alívio da dor é necessário, além da prescrição de analgesia, a adoção de medidas não farmacológicas, abordando os aspectos psicossociais e espirituais. $\mathrm{O}$ alívio da dor foi considerado por todas as categorias profissionais um aspecto fundamental no cuidado ao paciente em paliação, conforme relatos:

Ausência de dor. Ele pode ter tudo, mas se ele não tiver dor, na minha concepção, eu acho que a gente está conseguindo atingir o objetivo determinado. F08

Deixar o paciente confortável, tirar dor, tirar as angústias. E10

Os profissionais também enfatizaram a promoção do conforto direcionado às necessidades físicas para alívio de sintomatologias comuns ao paciente em CP, como dispneia e náuseas, terapia nutricional adequada, auxílio respiratório e uso de sedação para desconfortos ventilatórios difíceis de controlar:

Necessidade de aliviar a dor, dor ele não pode sentir de jeito nenhum, desconforto também não, então, às vezes colocar uma sedaçâo continua para paciente que tem problema respiratório. M13

Vai desde abordagem de facilitar a parte principalmente respiratória dele, para que ele não tenha 
dificuldade, até o uso, quando necessário, de ventilação invasiva, para dar conforto respiratório. FO7

Alguns participantes enfatizaram a suspensão de procedimentos para melhorar o conforto do paciente em CP e evitar intervençóes dolorosas. Ressaltaram essa conduta médicos, enfermeiros e fisioterapeutas, conforme as falas:

A gente dá só o suporte básico de vida, não entra com droga vasoativa, não entra com antibiótico, não dialisa, não oferece dieta, não colhe exames. M05

A gente deixa de ser mais invasivo. As medicaçóes váo sendo mais espaçadas, a gente tenta priorizar $o$ que realmente traz o conforto. $\boldsymbol{E} \mathbf{1 1}$

O paciente entrou em paliação, é conforto! A gente náo mexe na questão da oxigenoterapia, dos parâmetros ventilatórios. Busca melhor forma possivel não interferir, não prolongar aquilo. FO2

Os participantes acreditam que evitar procedimentos invasivos, exames e medicaçóes que não modificarão o curso da doença são formas de reduzir desconfortos. A tomada de decisão relacionada ao processo de trabalho que envolve o CP são medidas utilizadas para melhorar o conforto.

\section{Proporcionando conforto para alcançar paz, dignidade e respeito}

Os profissionais, ao informar como atuam na promoção de conforto do paciente terminal, reconhecem a necessidade de garantir dignidade e respeito no processo de morte e morrer destes:

Quando o paciente está em CP, é direito dele a morte digna, é direito dele morrer entre os iguais, não ser mais um número, um leito, é ser o pai, a mãe, o filho. $\mathbf{E 0 8}$

Os participantes enfatizaram medidas assistenciais promotoras de conforto, com o intuito de proporcionar experiência de paz aos pacientes e família, através do controle da ansiedade e inquietaçôes, abordagem do medo e suas preocupaçôes:
Às vezes, as pessoas estão esperando alguém que as escute, às vezes, ansiedade ou medo é isso. Procurar ouvir, falar, não mentir nas informaçôes, passar calma e tranquilidade. TE03

As categorias profissionais, principalmente a psicóloga, fisioterapeutas, enfermeiros e nutricionistas expuseram a importância do conforto psicológico, percebido através do diálogo relacionado aos medos nessa fase, demonstração de carinho, atenção, palavras de coragem e força, conforme depoimentos:

Direcionar o cuidado para o conforto do paciente, às vezes, é disponibilizar um horário de visita maior. É um paciente que tem milhóes de dias internados na UTI e nunca mais foi do lado de fora ver o sol, então, a gente pode botar na cadeira, avaliar com a equipe. Tudo que possa fazer que deixe ele mais tranquilo, que ele sofra menos, eu acho que é valido. E07

O principal foco é trazer conforto, não é nutrir, tendo em vista que a conduta nutricional não vai alterar o prognóstico desse paciente. Nossa terapêutica não vai aumentar os dias de vida do paciente, mas sim, dar vida aos dias do paciente. NO3

Requerem um pouco mais da sua atenção, um pouco mais do carinho, do toque, das palavras. TE06

O conforto psicológico é oferecido, também, por meio da disponibilização de atividades de lazer e alimentação, de acordo com o desejo do paciente, foram intervençóes percebidas pelos profissionais como ferramentas para alcançar experiência de dignidade e respeito.

\section{Aproximação com entes queridos e fé como estratégia de conforto}

Os participantes revelaram que valorizam a aproximação da família durante a fase terminal, representado pela extensão da visita em tempo integral. A criação de vínculo como instrumento de proporcionar segurança para paciente/família e o apoio espiritual para o alívio do sofrimento foram algumas alternativas sugeridas na promoção de conforto na UTI: 
As açóes são fazer algum estudo de caso com eles, conversando com eles, fazer orientaçóes, reunióes com a familia, para entender esse paciente e o contexto familiar dele. ASO1

A familia demonstrou ansiedade ou uma angústia? Então, eu abordo sempre pela questão da fé. Que tem que se manter a fé, buscar a religiāo dela, seja ela qual for. $\mathbf{F O C}$

A gente oferece a assistência religiosa. Aqui no hospital existe um capelão, mas quando essa familia não é católica, a gente oferece para eles a possibilidade de trazer alguém de fora que tenha uma importância para esse paciente. PO1

A aproximação dos familiares durante a internação foi percebida como mecanismo de redução de fatores ambientais hostis, destacando-se alguns cuidados para tornar o ambiente mais confortável:

Às vezes, trazer alguma coisa que lembre a casa, algum objeto religioso. Eles gostam de trazer terços, santinhos, cristais, então, eu flexibilizo. E07

Durante o atendimento, iluminar a sala com luz natural talvez seria uma ação interessante para tornar o ambiente um pouco mais humano. F08

Em virtude das características e invasividade da UTI, os participantes propóem alternativas para melhorar o ambiente e aproximar a família do cuidado.

\section{Discussão}

Apesar do presente estudo ter como limitaçóes à realização em apenas uma UTI de hospital privado, restringindo a um universo que pode não representar a realidade de outras equipes, sinaliza a necessidade de desenvolver um planejamento da assistência que favoreça o conforto holístico. Todos os profissionais relataram o conforto como elemento essencial do CP na UTI. A TFVP propóe que o paciente experimente conforto. ${ }^{(3)}$
Os resultados encontrados ultrapassam a lógica de uma teoria preditiva de médio alcance, visto que os profissionais demonstraram utilizar práticas assistenciais para promoção de um fim de vida pacífico que não estáo padronizados em protocolos. A percepção da equipe multiprofissional sobre CP corrobora com os conceitos da TFVP, sugerindo a identificação das necessidades básicas de cada paciente e discussão multiprofissional para construção de um plano de cuidados, pautado em evidências científicas, direcionado para a promoção do conforto. Criar estratégias de intervenção para o alivio da dor, aproximação com entes queridos, promoção de paz/dignidade/respeito e valorização da fé são dimensóes do cuidado que possibilitam um final de vida pacífico e que poderão ser utilizadas como protocolo de atuaçáo da equipe de enfermagem em estudos futuros.

Estudo qualitativo realizado em uma UTI de Salvador, Bahia, Brasil demonstrou que a promoção do conforto significa alívio de desconfortos físicos, como dor e angústia respiratória; oferta de suporte social e emocional à pessoa e família; garantia de medidas de higiene e prevenção de lesóes na pele. Assegurar essa integridade contribui para evitar desconforto físico, emocional e social. ${ }^{(10)}$

Estudo realizado com enfermeiros no Rio de Janeiro, Brasil, identificou que o alívio da dor e a promoção de conforto foram interpretados como sinônimos no âmbito dos CP, porém, compreender que a promoção do bem-estar não se limita ao controle da dor é fundamental para oferecer um cuidado multidimensional. Trata-se de diferentes conceitos da TFVP e de ferramentas para o desenvolvimento de $\mathrm{CP}$, complementando-se ou influenciando uma a outra. ${ }^{(11)}$

Os participantes referem que evitar a realização de procedimentos invasivos, exames e medicaçóes que não modificarão o curso da doença são formas de reduzir desconfortos na terminalidade. Essas medidas contribuem para melhoria da QV dos pacientes, sendo um dos objetivos da TFVP. ${ }^{(3)}$ Reconhecer o momento em que o tratamento é considerado fútil influencia diretamente na condução do cuidado na UTI. Essa etapa de planejamento e tomada de decisão é considera complexa pelos envolvidos, di- 
recionando para a necessidade de protocolos organizacionais específicos que proporcionem segurança nesse processo. ${ }^{(12,13)}$

Estudo realizado em Londres identificou quatro diferentes trajetórias de abordagem de CP: 1 . Cuidado curativo desde a admissão; 2. Oscilação entre cuidado curativo e de conforto; 3. Mudar definitivamente para o cuidado de conforto; 4. Cuidado de conforto desde admissão. O conflito ocorreu mais comumente nas trajetórias do padrão dois. As famílias aceitavam melhor o padrão três. ${ }^{(12)}$ Várias questóes éticas envolvem esse momento de decisão, na maioria dos casos, centralizado na figura médica, a qual pode fornecer comunicação, educação e discussão entre o paciente e família. ${ }^{(13)}$

O conforto é alcançado, também, pelas relações gentis que expressam tranquilidade e compreensão entre profissionais e paciente/família. A família se sente segura com o atendimento às suas necessidades, informaçóes claras e verdadeiras e a convicção de que seu parente está recebendo atendimento qualificado, do ponto de vista farmacológico, tecnológico e humano. Isso significa que o conforto vai depender de práticas que valorizem a humanidade associada à racionalidade. ${ }^{(14,15)}$

Estudo realizado em uma UTI cardiológica evidenciou as principais competências na realização dos CP. Entre 46 competências destacou-se a promoção de ambiente privativo e seguro; reconhecimento e apoio das necessidades familiares e conforto físico. Apenas uma citação estava relacionada às necessidades espirituais. ${ }^{(16)}$

Contrapondo os autores acima, algumas categorias profissionais deste estudo enfatizaram medidas para proporcionar conforto emocional, através da espiritualidade. Estudo realizado no sul do Brasil evidenciou que, recorrendo a espiritualidade, é possível oferecer conforto aos pacientes, através da criação de vínculo e incentivo à fé, obtendo uma resposta benéfica na ressignificação da finitude. ${ }^{(17,18)}$

A equipe de enfermagem foi a que mais se aproximou do cuidado holístico, evidenciando preocupação não só com o conforto físico, mas emocional, social e espiritual. O diagnóstico de conforto prejudicado pode ser um agravante ao paciente internado na UTI, sendo o enfermeiro o principal responsável por avaliar e implementar medidas prezando o bem -estar multidimensional, por estar mais próximo e ter o cuidado como instrumento do seu trabalho. ${ }^{(19)}$

A morte digna e pacífica, aquela que reúne os cinco elementos da TFVP deve ser uma prioridade para o planejamento de políticas e gerenciamento das UTIs. Discussóes sobre o prognóstico do paciente e tomada de decisão de forma precoce com a família, plano de cuidados holísticos, preparação da família e equipe para o processo de morrer e um ambiente privativo e construtivo definem o cenário para um fim de vida digno. ${ }^{(20)}$ Os participantes desta pesquisa citam essas medidas como ferramentas de promoção de conforto realizada por eles.

A UTI é um ambiente complexo e temido. A internação nela é associada a fatores negativos, destacando-se limitações físicas, falta de privacidade, iluminação incômoda, ruídos constantes, tecnologia dura com aparelhagens e distanciamento familiar. A maioria dos estressores são inalteráveis, pois representam suporte necessário para recuperação dos pacientes. Uma alternativa possível para construção de um ambiente mais confortável é o investimento em relaçôes de apoio, confiança e ética entre os membros da equipe e pacientes, a partir de atitudes simples como escuta sensível, acolhimento e ambiência. $(21,22)$ Outras alternativas relatadas pelos participantes é aproximar a família, através da visita estendida, trazer pertences dos pacientes, lazer e iluminação.

A promoção do conforto, desde a decisão pelos $\mathrm{CP}$ até o luto familiar, torna-se um imperativo moral diante desta vivência. ${ }^{(22)} \mathrm{Na}$ UTI, espera-se que seja o foco primordial dos CP. O conforto, na TFVP é sustentado por três pilares: alívio do desconforto, relaxamento e satisfação. Essas premissas são essenciais na busca pela $\mathrm{QV}$, possibilitando um bem-estar pleno. ${ }^{(3)}$ Neste contexto, a equipe multiprofissional deve ser capaz de prestar uma assistência pautada em açóes que priorizem o conforto, garantindo dignidade e respeito no processo de finitude.

\section{Conclusão}

O conforto foi o conceito da TFVP que se destacou na percepção da equipe multiprofissional, sendo pro- 
movido por todas as categorias na sua prática assistencial a pacientes terminais na UTI, motivado pela identificação das necessidades básicas destes, sejam inseridas no conforto físico, psicológico, ou promovido pela própria modificação das condiçóes ambientais. À luz da TFVP, observa-se que o conforto foi associado aos demais conceitos para promoçáo do fim de vida pacífico, reforçando a possibilidade de uma assistência pautada em teorias. A equipe de enfermagem foi a que mais se aproximou do conforto holístico, destacando outras dimensóes como a espiritual. Entretanto, o conforto proporcionado por meio do alívio da dor e de sintomas físicos foi o mais citado pelos profissionais, sinalizando a necessidade de capacitação que desperte a promoção de forma multidimensional. Intervençóes multiprofissionais pautadas nos conceitos da teoria podem ser utilizadas em outros serviços de paliação, auxiliando na busca por um fim de vida pacífico. Esse estudo contribui com o conhecimento no campo dos CP e da TFVP, ao direcionar os cuidados oferecidos na promoçáo do conforto a pacientes terminais na UTI, implicando em ações futuras que busquem a sistematização de CP baseada na identificação das necessidades básicas de cada paciente. Reforça-se, ainda, a importância de realização de novas pesquisas na área, para ampliar discussôes sobre a temática e TFVP.

\section{Colaborações}

Pires IB, Menezes TMO, Cerqueira BB, Albuquerque RS, Moura HCGB, Freitas RA, Santos ALS e Oliveira ES contribuíram com a concepção do estudo, análise e interpretação dos dados, redação do artigo, revisão crítica relevante do conteúdo intelectual e aprovação da versão final a ser publicada.

\section{Referências}

1. Pessini L, Siqueira JE. Reflections on care for critical patients at the end of life. Rev Bioética. 2019;27(1):29-37.

2. World Health Organization (WHO). Palliative care. Genève: WHO; 2018.

3. Ruland CM, Moore SM. Theory Construction based on standards of care: a proposed theory of the peaceful end of life. Nurs Outlook. 1998;46(4):169-75.
4. Fawcett J. Contemporary nursing knowledge: analysis and evaluation of nursing models and theories. 3rd ed. Philadelphia (US): F.A. Davis Company; 2013.

5. Wakiuchi J, Salimena AM, Sales CA. Being cared by a family member: the existential feelings of cancer patients. Texto Contexto Enferm. 2015; 24(2):381-9.

6. Zaccara AA, Costa SF, Nóbrega MM, França JR, Morais GS, Fernandes MA. Análise e avaliação da teoria final de vida pacífico segundo critérios de Fawcett. Texto Contexto Enferm. 2017; 26(4):e2920017.

7. Tong A, Sainsbury P, Craig J. Consolidated criteria for reporting qualitative research (COREQ): a 32-item checklist for interviews and focus groups. Int J Qual Health Care. 2007;19(6):349-57.

8. Bardin L. Análise de conteúdo. [Tradução de] Luís Augusto Pinheiro. São Paulo: Edições 70; 2016.

9. Conselho Nacional de Saúde. Resolução no 466 de 12 de dezembro de 2012. Aprova as diretrizes e normas regulamentadoras de pesquisas envolvendo seres humanos [Internet]. Brasília (DF): Conselho Nacional de Saúde; 2012 [citado 2019 Mar 29]. Disponível em: http://conselho. saude.gov.br/resolucoes/2012/Res0466.pdf.

10. Silva RS, Pereira A, Mussi FC. Comfort for a good death: perspective nursing staff's of intensive care. Esc Anna Nery. 2015;19(1):40-6.

11. Morais EM, Conrad D, Mattos EM, Cruz SA, Machado GC, Abreu MO. Palliative care: coping nurses in a private hospital in the city of Rio de Janeiro - RJ. J Res Fundam Care Online. 2018;10(2):318-25.

12. Santos EC, Oliveira IC, Feijão AR. Validation of a nursing care protocol for patients undergoing palliative care. Acta Paul Enferm. 2016 ;29(4):363-73.

13. Silva Junior SV, Silva TN, Freire ME, Santos LB. Palliative care to elderly hospitalized: discourses of nurse clinicians. Enferm Atual. 2019;(87):1-7.

14. Higginson IJ, Rumble C, Shipman C, Koffman J, Sleeman KE, Morgan $\mathrm{M}$, et al. The value of uncertainty in critical illness? An ethnographic study of patterns and conflicts in care and decision-making trajectories. BMC Anesthesiol. 2016;16(11):1-11.

15. Karnik S, Kanekar A. Ethical issues surrounding end-of-life care: a narrative review. Healthcare. 2016;4(24):1-6.

16. Fateel EE, O'Neill CS. Family members' involvement in the care of critically ill patients in two intensive care units in an acute hospital in Bahrain: The experiences and perspectives of family members' and nurses' - A qualitative study. Clin Nurs Stud. 2016;4(1):57-69.

17. Brabo BC, Laprano MG. Nurse's skills for cardiology palliative care. Rev Enferm UFPE Online. 2018;12(9):2341-48.

18. Arrieira IC, Thofehrn MB, Porto AR, Moura PM, Martins CL, Jacondino MB. Spirituality in palliative care: experiences of an interdisciplinary team. Rev Esc Enferm USP. 2018;52:e03312.

19. Barbosa RT, Cruz IC. [Nursing evidence-based interprofessional practice guidelines for impaired comfort related noise in ICU systematic literature review]. J Special Nurs Care. 2019;11(1):1-10. Portuguese.

20. Timmins F, Parissopoulos S, Plakas S, Naughton MT, Vries JM, Fouka G. Privacy at end of life in ICU: A review of the literature. J Clin Nurs. 2018;27(11-12):2274-84.

21. Gomes AG, Carvalho MF. TThe patient's perspective regarding hospitalization experience in intensive care unit (ICU): integrative literature review]. Rev SBPH. 2018;21(2):167-85. Portuguese.

22. Valente CO, Fonseca GM, Freitas KS, Mussi FC. Family comfort to a relative in the intensive therapy unit. Rev Baiana Enferm. 2017;31(2):1-9. 